\section{3- vs 20-gauge pars plana vitrectomy in combination with bimanual microincisional cataract surgery (b-MICS) for the treatment of macular hole and cataract as a one-step procedure}

C-L Schönfeld
Augenklinik Herzog Carl Theodor, München, Germany

\section{Correspondence:}

C-L Schönfeld, Augenklinik Herzog Carl Theodor, Nymphenburger Street 43, München, 80335, Germany Tel: + 4989 1270930; Fax: + 4989 1290341; E-mail: cb.schoenfeld@ gmx.net

Received: 9 August 2012 Accepted in revised form: 9 April 2013

Published online: 7 June 2013

\begin{abstract}
Background/aims 23-gauge pars plana vitrectomy (ppv) is a new method for vitreoretinal surgery. It may reduce operation time, the risk of complications, and patient discomfort, especially in combination with simultaneous bimanual microincisional cataract surgery (b-MICS).

Methods Seventy-five consecutive patients who underwent combined cataract surgery and ppv at our center between 1 January 2008 and 31 December 2010 were included. The first 36 patients were treated with 20-gauge ppv, the following 39 patients with 23-gauge ppv. Study end points 8 weeks after surgery were duration of the procedure, improvement of visual acuity, and occurrence of complications.

Results Duration of surgery was reduced in 23- vs 20-gauge ppv (54.0 \pm 11.6 vs $61.0 \pm 19.0 \mathrm{~min}, P=0.08)$. Visual acuity improved significantly in both the groups (20-gauge: logMAR $0.750 \pm 0.304$ before and $0.369 \pm 0.273$ after surgery; 23-gauge: $\log$ MAR $0.663 \pm 0.340$ before and $0.339 \pm 0.273$ after surgery). There were no appreciable group differences in baseline or post-treatment visual acuity.

Conclusions 23-gauge ppv in combination with b-MICS is a suitable, safe, and effective method for the treatment of combined cataract and vitreo-retinal diseases. The
\end{abstract}

procedure is somewhat shorter and patient discomfort during and after surgery is improved. In terms of efficacy and safety, 23-gauge ppv is equivalent with conventional 20-gauge ppv.

Eye (2013) 27, 952-958; doi:10.1038/ eye.2013.118; published online 7 June 2013

Keywords: macular hole; vitrectomy; micro incision; 23 gauge; cataract surgery

Introduction

Pars plana vitrectomy (ppv) was introduced in the 1970s as a method for the removal of an opaque vitreous, ${ }^{1}$ and the indication for surgery has substantially broadened since as a consequence of a multitude of technical advancements.

Contemporary indications for ppv include retinal detachment, epiretinal membranes, macular holes, diabetic retinopathy, vitreous hemorrhages, proliferative vitreoretinopathy, retinal vein occlusion, and rare vitreous and retinal pathologies. All these pathologies coexist with cataract formation. Consequently, combined vitreo-retinal and cataract surgery has been established during the 1980s and 1990s and is under discussion up to date..$^{2-4}$

One of the aforementioned technical developments is the employment of smallcaliber instruments first described by Fujii et $a l^{5}$ 
in 2002. For the first approximately three decades, ppv was usually performed with 20-gauge instruments that were inserted into the vitreous via a sclerotomy through the pars plana; ${ }^{6}$ the resulting scleral cut typically requires surgical sealing with sutures. By contrast, smallgauge instruments can be inserted in the vitreous through a so-called 'port' and typically do not require suturing. Another advantage of the small-gauge vitrectomy is the reduced surgical trauma, but the approach has also caliber-related disadvantages such as a longer duration of vitreous extraction, less stability of the instrument itself, and poorer illumination of the retina. 6,7

It has been proposed that small-gauge vitrectomy reduces patient discomfort and the risk of inflammatory or infectious complications; consequently, the acceptance and clinical application has increased considerably over the past decade. ${ }^{6}$ However, the evidence for such benefits is inconsistent, ${ }^{8}$ and the practical realization of potential advantages is subject to a number of important confounding factors, most prominently the surgical skill and experience of the surgeon. ${ }^{9}$ It is proposed that certain interventions - especially more extensive procedures—still require standard 20-gauge instruments, ${ }^{6}$ and moreover, 20-gauge ppv is doubtlessly still the 'gold standard' against which technical and surgical innovations have to be measured in terms of efficacy and safety.

Ppv in combination with extracapsular cataract extraction has traditionally been performed as a two-step procedure, but the single-session surgery appears to be equally effective. ${ }^{4}$ However, a reduced inflammatory reaction is considered to be an advantage of the two-step approach, ${ }^{4}$ and therefore, small-gauge ppv is of particular interest for the one-step combination of vitreoretinal and cataract surgery.

A number of authors performed combined operations with increasing frequency, and the introduction of microincisional cataract surgery (MICS) ${ }^{10}$ was paralleled by technical improvements of the 23-gauge instruments. Therefore, the clinical application of 23-gauge ppv was considered a promising alternative to the conventional 20-gauge ppv for combined cataract and vitreo-retinal surgery; this approach has not been evaluated systematically yet.

The present study investigates the feasibility, safety, and efficacy of 23-gauge ppv in patients with macular hole and cataract in comparison to the conventional 20-gauge ppv. Study end points were:

- the procedure's duration,

- the improvement of the visual acuity, and

- the incidence of complications after the procedure.

\section{Materials and methods}

\section{Patients}

Seventy-five consecutive patients who underwent combined one-step cataract surgery and ppv at our center between 1 January 2008 and 31 December 2010 were included in the analysis. The observation period was chosen because it covered the shift between 20gauge (36 patients, operated between 1 January 2008 and 31 August 2009) and 23-gauge (39 patients, operated between 1 September 2009 and 31 December 2010) ppv and provided virtually identical cohort sizes. There were no differences between the two groups with respect to their demographics (Table 1).

\section{Surgical procedures}

All surgical procedures were carried out by the author (C-LS.) who has extensive experience in vitreo-retinal surgery in excess of $7000 \mathrm{ppv}$ procedures. All operations were performed with the Millennium Microsurgical System (Bausch and Lomb Inc., Rochester, NY, USA). Pupils were dilated preoperatively using tropicamide (Pharma Stulln GmbH, Stulln, Germany) and phenylephrine $10 \%$ neosynephrine POS eyedrops alternately (twice each), applied from $1 \mathrm{~h}$ before surgery. Subsequently, the eyelids were disinfected with povidone $10 \%$ iodine solution and a gauze pad was left in place for at least $7 \mathrm{~min}$. A mixture of $5 \mathrm{ml}$ bupivacain $0.5 \%$ (Carbostesin) and $2 \mathrm{ml}$ mepivacain $1 \%$ (Scandicain) was used, and 75 I.U.Hyaluronidase (Hyalase) were added. Afterwards, the eyes were again covered with a gauze pad containing $10 \%$ povidone for $7 \mathrm{~min}$. The eyes were covered with sterile cloths, and the fornices were preoperatively rinsed with $1 \%$ povidone solution. In all the cases, bimanual MICS (b-MICS) was performed.

Table 1 Demographics of the two groups

\begin{tabular}{lcc}
\hline & 20-Gauge PPV & 23-Gauge PPV \\
\hline Observation period & 1 January 2008-31 August 2009 & 1 September 2009-31 December 2010 \\
Number of patients (\%) & $36(48.0 \%)$ & $39(52.0 \%)$ \\
Age (mean \pm SD) & $72.1 \pm 6.7$ years & $71.4 \pm 5.3$ years \\
Females/males & $20(55.6 \%) / 16(44.4 \%)$ & $22(56.4 \%) / 17(43.6 \%)$ \\
\hline
\end{tabular}


Two paracenteses with a width of $1.5 \mathrm{~mm}$ were placed at the 3 and 9 O'clock positions, respectively, using a disposable phacoknife (Alcon Laboratories, Hemel Hempstead, UK). An ocular viscosurgical device (sodium hyaluronate, Healon, AMO Inc., Santa Ana, CA, USA) was introduced, and a continuous anterior capsulotomy was performed using a 23-gauge cannula. The inner nucleus was hydrodissected, and a bimanual phacemulsification was performed. Due to mostly 'soft' lenses, the total duration of full ultrasound power application did not exceed $3 \mathrm{~s}$ in any of our cases. Cortex was removed using the bimanual irrigation aspiration system of the Millennium system (Bausch and Lomb). In all the patients, a hydrophilic, lipophilic coated acrylic lens (SM 409 m, Carl Zeiss Meditc AG, Oberkochen, Germany) was implanted under sodium hyaluronate (Healon, AMO Inc.) after enlarging the incision temporally to $2.0 \mathrm{~mm}$. After placement of the intraocular lens (IOL) into the capsule, sodium hyaluronate was removed above and below the IOL using the irrigation aspiration device. Then vitrectomy was performed. In 20gauge vitrectomy, the conjunctiva was opened radially using a single use MVR blade (Alcon Laboratories), and hemorrhages were stopped using the coagulation devices. Three sclerotomies were placed $3.5 \mathrm{~mm}$ from the limbus at 10:30, 1:30 and 4:30 clock positions, respectively. Subsequently, a suture was placed at the 4:30 sclerotomy using 7-0 vicryl (Ethicon Inc., Somerville, NJ, USA), and the infusion tube was secured with that suture. In 23-gauge vitrectomies, ports were transconjunctivally inserted using the Millennium trocar system (Bausch and Lomb). The trocars were inserted almost tangentially to the bulb and then turned inwards at an approximate $70^{\circ}$ angle to facilitate pressureinduced auto-closure. Firstly, an anterior vitrectomy was performed using the squint hook for indentation. Then, the Biom (Oculus, Wetzlar, Germany) was used to access the vitreous at the posterior pole. If necessary, posterior vitreous detachment was created using the cutter with high suction and nil cutting rate, and remnants of the anterior vitreous were removed without the Biom under higher magnification using the squint hook again.

In all the stages of macular holes, inner limiting membrane (ILM) peeling was performed. The ILM was selectively stained with a blue dye under balanced salt solution for about 5-10s. (Brilliant Peel, Geuder AG, Heidelberg, Germany). Immediately after the installation, the brilliant peel was removed with the cutter. The ILM was peeled under the highest microscope magnification using a disposable flexible plan concave lens (FCI Ophthalmics, Marshfield Hills, MA, USA) and a disposable end-gripping Eckardt forceps (Geuder AG) of a gauge-corresponding size. The ILM was gripped, lifted slightly, and turned in small circles and finally released. A small scissure appeared whose end was gripped and employed for a flap preparation. The ILM flap was pulled towards the fovea in a broad fold to facilitate non-touching of the retina at and around the fovea. At the end of the surgery, vitreous fluid was exchanged using a disposable fluid needle (Geuder AG), and gas was injected in 15\% (for 20 gauge) or $20 \%$ (for 23 gauge) concentrations, respectively. In 20-gauge vitrectomy, all sclerotomies were closed using 7-0 vicryl (Ethicon Inc.), and in 23-gauge vitrectomy trocars were withdrawn and no sutures placed. Each patient received a peribulbar administration of $75 \mathrm{mg}$ cefuroxim (Hexal AG, Holzkirchen, Germany) and $4 \mathrm{mg}$ dexamethason (Jenapharm, Jena, Germany). Ointment was given to all the patients using a mixture of dexamethason, neomycin, and polymyxin B (Isoptomax, Alcon Pharma, Freiburg, Germany) and the eyelids were closed using strapping. Gauze was placed on top of the eyelids and covered with an eye patch.

Postoperatively, a mixture of dexamethason, neomycin, and polymyxin B ointment was applied six times daily and tropicamide twice daily for 4 days. Intraocular pressure (IOP) was measured daily for 4 days and controlled to remain $\leq 30 \mathrm{~mm} \mathrm{Hg}$. If the IOP rose above that threshold, eyes were treated with antiglaucoma eye drops. After 5 days, the ointment was changed to drops that were given for another week. After 1 week, prednisolone acetate eye drops (Inflanefran forte, Allergan Inc., Irvine, CA, USA) were applied, and the dose was reduced to four times daily and by one further drop per subsequent week. Patients were monitored by their referring ophthalmologists and examined by the operating surgeon (C-LS) 8 weeks after surgery. No lenspupil captures occurred during the postoperative period.

\section{Evaluation}

General ophthalmological diagnostics were complemented by optical coherence tomography (OCT) scans and comprehensive registration of all related and unrelated complications during surgery and convalescence. The following variables were assessed and stored for statistical evaluation: Demographics, degree of macular hole, visual acuity $(\log M A R$ (logarithm of the minimal angle of resolution)) before and 8 weeks after the operation, duration of the procedure, and occurrence of complications and re-interventions.

\section{Statistical analysis}

All statistical analyses were performed with the SPSS software package (V. 15.0, SPSS Inc., Chicago, IL, USA). Non-parametric test methods were employed, and group 


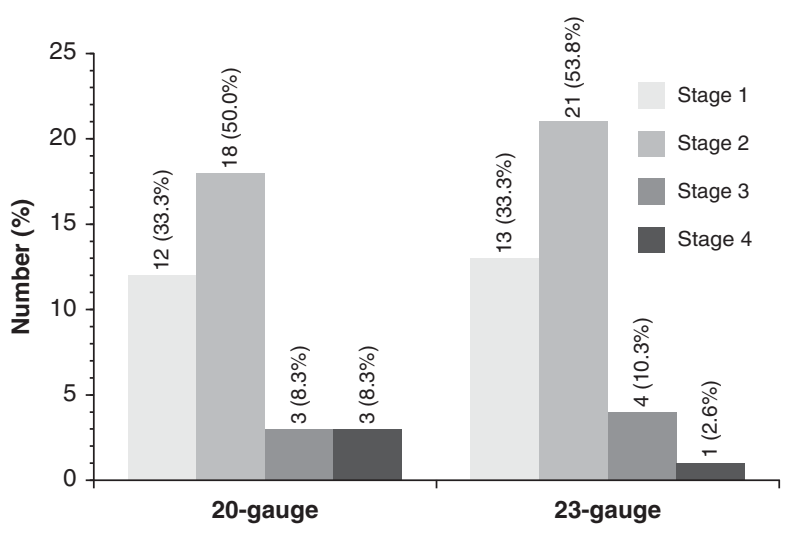

Figure 1 Macular hole stage at baseline in patients who underwent 20- or 23-gauge ppv, respectively.

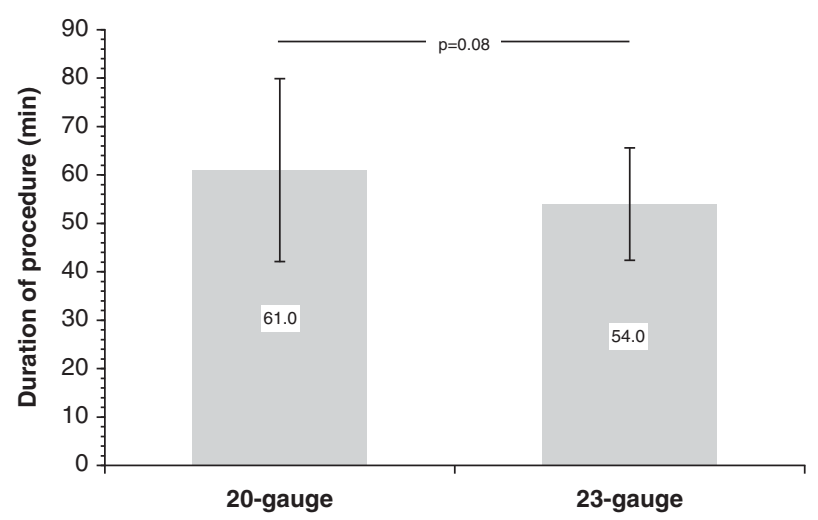

Figure 2 Duration of the surgical procedure in patients who underwent 20- or 23-gauge ppv, respectively.

differences with a $P$-value $<0.05$ were considered statistically significant.

I certify that all applicable institutional and governmental regulations concerning the ethical use of human volunteers were followed during this research.

\section{Results}

There was no appreciable group difference in the macular hole stage at baseline (Figure 1).

The operation time was appreciably (albeit, not significantly) shorter with 23-gauge vitrectomy, amounting to a difference of $7 \mathrm{~min}$ or $11.5 \%$ (Figure 2).

Baseline visual acuity showed no significant group difference, although the visual acuity was slightly better before 23-gauge ppv. There was a statistically significant improvement in both the groups, and neither the difference achieved by the surgery nor the values 8 weeks after the procedure were significantly different in the treatment groups (Figure 3).

All in all, complications were rare. Three patients from either group had to undergo a reoperation because of

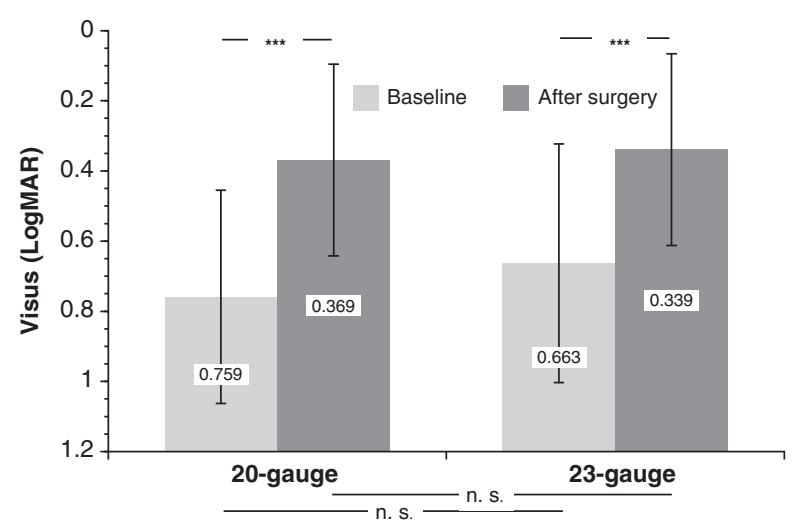

Figure 3 Visual acuity $(\log M A R)$ at baseline and 8 weeks after surgery in patients who underwent 20- or 23-gauge ppv, respectively. The $\mathrm{y}$-scale was adapted to visualize the visual improvement (NS, not significant, ${ }^{* * *} P<0.0001$ ).

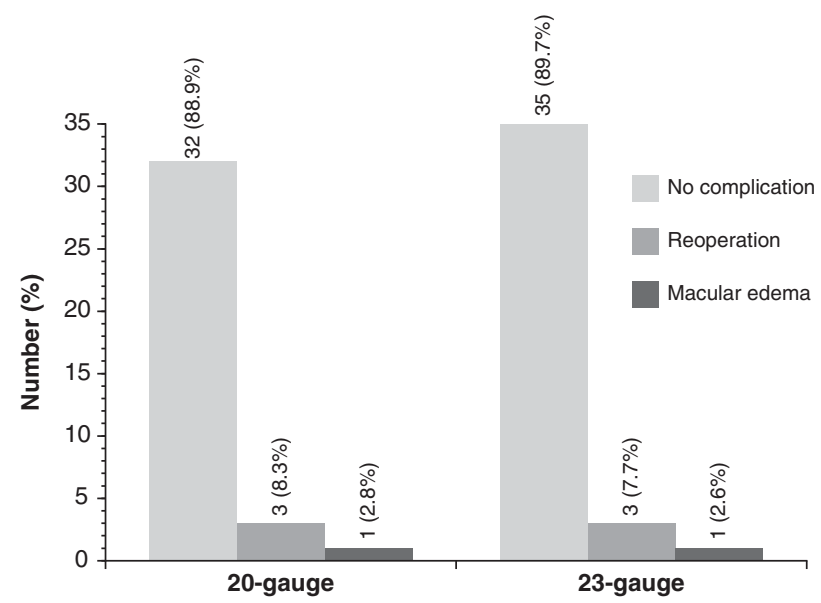

Figure 4 Re-interventions in patients who underwent 20- or 23-gauge ppv, respectively.

persistent macular hole, and one patient of each group received intra-vitreous dexamethasone (23 gauge) or triamcinolone (20 gauge), respectively, because of macular edema after ppv (Figure 4). There was no occurrence of endophthalmitis or persistent hypotonia.

\section{Discussion}

The present study clearly confirms the suitability of a small-gauge one-step approach to the surgical treatment of combined diseases of the lens and the retina. The longer duration of vitreous extraction through the smaller-caliber instrument is obviously more than outweighed by technical simplification with a net shortening of procedural time by about $10 \%$. Whereas some authors describe a shorter operation time for 23-7,11,12 and 25-gauge ppv, ${ }^{5,7,12}$ respectively, others find no difference. ${ }^{13,14}$ When comparing operation times, the exact procedure and its respective time requirement must be considered; for instance, a longer duration of the 
actual vitreous extraction that outweighs the benefit of the non-suture may be a result of a lower cutting rate. Moreover, the additional cataract operation performed in the present series must be accounted for about 10-15 min, putting the operation time in the present series more or less in the same order of magnitude as in most other trials. In general, surgical care is partly in contradiction with short procedure duration and should definitely enjoy a higher priority. All in all, the small-gauge ppv may not necessarily be shorter than conventional 20-gauge ppv, but the overall time demand of the procedure is certainly not higher.

In terms of safety, we found no difference between 20- and 23-gauge ppv and generally a low frequency of complications. A persistent macular hole required a re-intervention in about $8 \%$ of the sample, and there was only one case of postoperative macular edema. The excellent safety of ppv and the lack of a difference depending on the instrument caliber is in concordance with published evidence. ${ }^{6}$ The risk of postoperative hypotony was reported to be increased after non-sutured small-gauge ppv; $;, 15,16$ in our series, no such case occurred, possibly as a consequence of the higher concentration of the $\mathrm{C}_{2} \mathrm{~F}_{6}$ gas (20\% as compared with $15 \%$ ) used for the tamponade and/or the prone position of the patient, both of which have proven to be effective previously. ${ }^{17}$ Therefore, the suture recently suggested by Kusuhara et al ${ }^{18}$ seems to be unnecessary in most cases.

The most dreaded complication in ocular surgery, endophthalmitis, was not encountered in either group of the present study. It has been suggested that endophthalmitis may occur more frequently after smallgauge interventions, possibly due to migration of bacteria through the open sclerotomy injuries. 7,19,20 Hypotonia may aggravate this problem because of a pressure gradient directed into the vitreous. However, methodically careful investigations failed to confirm a pertinent difference, ${ }^{6,8,21}$ and the incidence of endophthalmitis is well below $0.1 \%$ in most published series, ${ }^{6,8,21}$ meaning that in patient samples like that of the present trial only sporadic cases could have occurred. On the other hand, there are also reports of a much higher incidence, reaching up to $2.4 \% .^{22}$ Such a frequency should, in general, give rise to careful procedural evaluations and is hardly attributable to the caliber of the ppv instrument, and reports of significant group differences ${ }^{19,20}$ ought to be carefully scrutinized concerning methodical issues.

The outcome of combined ppv and phacoemulsification in terms of the visual acuity was very satisfactory in the present trial, and there was no appreciable difference between 20- and 23-gauge ppv; once again, these results confirm previously published outcome data. ${ }^{6,11,12,23}$ All in all, single-session 23-gauge ppv with b-MICS yields excellent results equivalent to those of conventional twostep procedures in the treatment of macular hole with cataract.

From a methodological point of view, the present study is somewhat limited because it is not prospectively randomized. However, the fact that all patients were operated by the same surgeon and the methods were switched completely in the middle of the observation period provide a data quality that is practically on par with a randomized trial. Possible sources of bias in the choice of methods are eliminated, and the samples show an excellent match in terms of demographics and baseline findings. The surgical treatment of stage 1 macular hole (exactly one-third of either group in the present trial) may give rise to some skepticism as far as the ophthalmological outcome is concerned. Although surgical treatment is recommended for stages 3 and 4 in general and for stage 2 because of the high risk of progression, surgical closure of stage 1 macular holes is practiced frequently when spontaneous closure fails to commence. ${ }^{24}$ Obviously, spontaneous closure of stage 1 macular holes cannot be taken for granted as all advanced forms have passed this stage initially, and on the other hand, spontaneous closure has been observed in advanced stages as well. ${ }^{25}$ In all cases where OCT reveals a pull on the outer retina by antero-posterior vitreous traction, a progression to higher stages of macular hole is highly likely, ${ }^{26}$ and surgical treatment is the logical consequence, in particular when a coexisting cataract is present.

At this point, it has to be emphasized that the goal of the present study is mainly the comparison between small- and regular-gauge ppv in combination with b-MICS and not so much the comparison of visual acuity outcome with that of other studies. The basic efficacy of ppv for the improvement of the visual acuity in patients with vitreo-retinal diseases has been established extensively and needs no further confirmation; moreover, a study with only 75 patients would have hardly augmented the already more than sufficient body of evidence based on much higher patient numbers.

Therefore, the surgical and ophthalmological outcome of 23-gauge ppv is at least on par with that of conventional 20-gauge ppv, and the advantages of this approach are quite substantial: In accordance with our personal observation, patients experience less discomfort during and after the procedure, less irritation and fewer conjunctival edema, decreased doses of antiinflammatory medication, a shorter duration of postoperative treatment, and a faster overall recovery. 6,14 Therefore, ophthalmologists should be encouraged to train and practice the small-gauge ppv.

The choice between 23-, 25-, and 27-gauge ppv is presently mainly based on the surgeon's preference and 
availability/economy of instruments. Obviously, the advantages of small-gauge ppv are increasing with smaller calibers of the instruments. On the other hand, the disadvantages - that is, less stability of the instruments and consequent difficulty in the assessment of the distance to the retina, requirement of higher cutting rates-also increase, making the learning curve for 25- and especially for 27-gauge ppv somewhat steeper than that for 23-gauge ppv. The relevance of the learning curve becomes obvious under consideration of relative frequent complications in earlier published series. $^{15}$

Therefore, presently the 23-gauge ppv seems to be a good compromise between the more traumatic 20-gauge and the technically more demanding 25- or 27-gauge ppv.

\section{Summary}

\section{What was known before}

- 20-gauge ppv macular surgery combined with cataract surgery is not a standard procedure. Reasons are patient discomfort, duration of the procedure, and surgical trauma.

\section{What this study adds}

- 23-gauge ppv combined with b-MICS reduces operation time and surgical trauma. Results are comparable and potentially better.

\section{Conflict of interest}

The author declares no conflict of interest.

\section{Acknowledgements}

This research received no specific grant from any funding agency in the public, commercial or not-for-profit sectors. C-LS has full control of all the primary data.

\section{References}

1 Machemer R, Buettner H, Norton EW, Parel JM. Vitrectomy: a pars plana approach. Trans Am Acad Ophthalmol Otolaryngol 1971; 75: 813-820.

2 Chaudhry NA, Cohen KA, Flynn Jr., HW, Murray TG. Combined pars plana vitrectomy and lens management in complex vitreoretinal disease. Semin Ophthalmol 2003; 18: 132-141.

3 Demetriades AM, Gottsch JD, Thomsen R, Azab A, Stark WJ, Campochiaro PA et al. Combined phacoemulsification, intraocular lens implantation, and vitrectomy for eyes with coexisting cataract and vitreoretinal pathology. $A m \mathrm{~J}$ Ophthalmol 2003; 135: 291-296.

4 Treumer F, Bunse A, Rudolf M, Roider J. Pars plana vitrectomy, phacoemulsification and intraocular lens implantation. Comparison of clinical complications in a combined versus two-step surgical approach. Graefes Arch Clin Exp Ophthalmol 2006; 244: 808-815.

5 Fujii GY, De Juan Jr, E, Humayun MS, Pieramici DJ, Chang TS, Awh C et al. A new 25-gauge instrument system for transconjunctival sutureless vitrectomy surgery. Ophthalmology 2002; 109: 1807-1812; discussion 1813.

6 Recchia FM, Scott IU, Brown GC, Brown MM, Ho AC, Ip MS. Small-gauge pars plana vitrectomy: a report by the American Academy of Ophthalmology. Ophthalmology 2010; 117: 1851-1857.

7 Spirn MJ. Comparison of 25, 23 and 20-gauge vitrectomy. Curr Opin Ophthalmol 2009; 20: 195-199.

8 Shimada H, Nakashizuka H, Hattori T, Mori R, Mizutani Y, Yuzawa M. Incidence of endophthalmitis after 20- and 25 -gauge vitrectomy causes and prevention. Ophthalmology 2008; 115: 2215-2220.

9 Ramkissoon YD, Aslam SA, Shah SP, Wong SC, Sullivan PM. Risk of iatrogenic peripheral retinal breaks in 20-G pars plana vitrectomy. Ophthalmology 2010; 117: 1825-1830.

10 Kohnen T. MICS-Mikroinzisionale Kataraktchirurgie. Ophthalmologe 2010; 107: 105-107.

11 Hikichi T, Matsumoto N, Ohtsuka H, Higuchi M, Matsushita T, Ariga $\mathrm{H}$ et al. Comparison of one-year outcomes between 23- and 20-gauge vitrectomy for preretinal membrane. Am J Ophthalmol 2009; 147 (639-643): e631.

12 Sandali O, El Sanharawi M, Lecuen N, Barale PO, Bonnel S, Basli E et al. 25-, 23-, and 20-gauge vitrectomy in epiretinal membrane surgery: a comparative study of 553 cases. Graefes Arch Clin Exp Ophthalmol 2011; 249: 1811-1819.

13 Albrieux M, Rouberol F, Bernheim D, Romanet JP, Chiquet C. Comparative study of 23-gauge vitrectomy versus 20-gauge vitrectomy for the treatment of rhegmatogenous retinal detachment. Graefes Arch Clin Exp Ophthalmol 2011; 249: 1459-1468.

14 Wimpissinger B, Kellner L, Brannath W, Krepler K, Stolba $\mathrm{U}$, Mihalics $\mathrm{C}$ et al. 23-Gauge versus 20-gauge system for pars plana vitrectomy: a prospective randomised clinical trial. Br J Ophthalmol 2008; 92: 1483-1487.

15 Gupta OP, Ho AC, Kaiser PK, Regillo CD, Chen S, Dyer DS et al. Short-term outcomes of 23-gauge pars plana vitrectomy. Am J Ophthalmol 2008; 146: 193-197.

16 Von Fricken MA, Kunjukunju N, Weber C, Ko G. 25-Gauge sutureless vitrectomy versus 20-gauge vitrectomy for the repair of primary rhegmatogenous retinal detachment. Retina 2009; 29: 444-450.

17 Malik A, Dooley I, Mahmood U. Single night postoperative prone posturing in idiopathic macular hole surgery. Eur J Ophthalmol 2012; 22(3): 456-460.

18 Kusuhara S, Ooto S, Kimura D, Itoi K, Mukuno H, Miyamoto $\mathrm{N}$ et al. Intraocular gas dynamics after 20-gauge and 23-gauge vitrectomy with sulfur hexafluoride gas tamponade. Retina 2011; 31: 250-256.

19 Chen JK, Khurana RN, Nguyen QD, Do DV. The incidence of endophthalmitis following transconjunctival sutureless 25- vs 20-gauge vitrectomy. Eye (Lond) 2009; 23: 780-784.

20 Kunimoto DY, Kaiser RS. Incidence of endophthalmitis after 20- and 25-gauge vitrectomy. Ophthalmology 2007; 114: 2133-2137.

21 Scott IU, Flynn Jr HW, Acar N, Dev S, Shaikh S, Mittra RA et al. Incidence of endophthalmitis after 20-gauge vs 23-gauge vs 
25-gauge pars plana vitrectomy. Graefes Arch Clin Exp Ophthalmol 2011; 249: 377-380.

22 Haas A, Seidel G, Steinbrugger I, Maier R, Gasser-Steiner V, Wedrich A et al. Twenty-three-gauge and 20-gauge vitrectomy in epiretinal membrane surgery. Retina 2010; 30: 112-116.

23 Narayanan R, Sinha A, Reddy RK, Krishnaiah S, Kuppermann BD. Faster visual recovery after 23-gauge vitrectomy compared with 20-gauge vitrectomy. Retina 2010; 30: $1511-1514$
24 Subramanian ML, Truong SN, Rogers AH, Duker JS, Reichel E, Baumal CR. Vitrectomy for stage 1 macular holes identified by optical coherence tomography. Ophthalmic Surg Lasers Imaging 2006; 37: 42-46.

25 Schweitzer KD, Garcia R. Spontaneous closure of a stage III idiopathic macular hole. Can J Ophthalmol 2007; 42: 127-128.

26 Takahashi A, Nagaoka T, Ishiko S, Kameyama D, Yoshida A. Foveal anatomic changes in a progressing stage 1 macular hole documented by spectral-domain optical coherence tomography. Ophthalmology 2010; 117: 806-810. 